\title{
Assessment of the sedative effects of dexmedetomidine and propofol treatment in patients undergoing mechanical ventilation in the ICU and relationship between treatment and occurrence of ventilator-associated pneumonia and detection of pathogenic bacteria
}

\author{
HONGJIE DOU, FANGBAO HU, WEN WANG, LIN LING, DEQIANG WANG and FENLIAN LIU \\ Intensive Care Unit, Shanghai Fengxian District Central Hospital, Fengxian, Shanghai 201499, P.R. China
}

Received June 10, 2019; Accepted February 13, 2020

DOI: $10.3892 /$ etm.2020.8699

\begin{abstract}
The present study aimed to investigate the sedative effects of dexmedetomidine combined with propofol in patients undergoing mechanical ventilation in the intensive care unit (ICU), and to reveal the risk factors of ventilator-associated pneumonia (VAP). A retrospective analysis of 322 patients who had been subject to mechanical ventilation in the ICU ward was performed. Subjects were divided into two groups: A group treated with dexmedetomidine and propofol (combined group) and a group treated with dexmedetomidine alone (monotherapy group). Clinical data, sedative effects, the number of VAP patients and the distribution of VAP pathogens were assessed. Multivariate analysis and receiver operating characteristic (ROC) curves were used to predict VAP. Significant differences in the sedative effects between the two groups were observed $(\mathrm{P}<0.001)$. The incidence of VAP was significantly higher in the monotherapy group compared with the combined group $(\mathrm{P}<0.05)$. Multivariate logistic regression analysis demonstrated that age, acute physiology chronic health evaluation score, consciousness, invasive operations, recovery time, extubation time and sedation regimen were independent risk factors for VAP in the ICU during mechanical ventilation. ROC curves indicated that the areas under the curve for age, acute physiology chronic health score, consciousness, invasive operations, recovery time, extubation
\end{abstract}

Correspondence to: Dr Fangbao $\mathrm{Hu}$, Intensive Care Unit, Shanghai Fengxian District Central Hospital, 6600 Nanfenggong Road, Fengxian, Shanghai 201499, P.R. China

E-mail: hufangbao428@126.com

Abbreviations: ICU, intensive care unit; VAP, ventilator-associated pneumonia; ROC, receiver operating characteristic; APACHE, acute physiology chronic health

Key words: dexmedetomidine, propofol, intensive care unit, mechanical ventilation, ventilator-associated pneumonia time and sedation regimen were 0.934, 0.870, 0.632, 0.677, $0.865,0.950$ and 0.603 , respectively. In summary, dexmedetomidine combined with propofol can shorten the recovery and extubation times of mechanical ventilation patients in the ICU. Different sedation schemes are also independent risk factors for VAP during mechanical ventilation in the ICU.

\section{Introduction}

Intensive Care Units (ICUs) treat critically ill patients in hospitals and tracheal intubation is typically required for assisted ventilation in ICU wards. Tracheal intubation primarily consists of mechanical ventilation, to which many patients develop intolerance $(1,2)$. Sedative and analgesic adjuvant therapy is often required during clinical treatment, which reduces the discomfort and stress responses of patients and increases the effectiveness of mechanical ventilation (3). Moreover, studies have shown that appropriate sedation can improve the clinical outcomes of patients (4). The most commonly used sedative in the clinic is dexmedetomidine (5). Dexmedetomidine, as a highly selective $\alpha 2$-adrenergic receptor agonist, inhibits the release of thyroxine by activating $\alpha 2$-adrenergic receptors, reducing sympathetic nervous system activity (6). Patients receiving dexmedetomidine anesthesia do not develop respiratory depression, which is conducive for those with difficulties weaning from mechanical ventilation (7). As a short-acting alkylphenol sedative and hypnotic drug, propofol acts on GABA receptors in the central nervous system and has the characteristics of rapid onset, a short half-life, fast metabolism and a lack of accumulation in the body (8). However, patients may experience memory loss and anti-convulsive effects after propofol use (9).

Ventilator-associated pneumonia (VAP) is a common cause of pulmonary infection in ICU wards. The incidence of pulmonary infections during mechanical ventilation in the ICU is much higher than that in other hospital departments (10). Artificial mechanical ventilation provides a direct route for pathogens to enter the body and damages the physical barrier of the airway, leading to reduced immune resistance, further increasing the risk of infection (11). When patients 
develop pulmonary infections, their condition deteriorates, affecting the treatment of primary diseases. Dexmedetomidine combined with propofol reduces extubation times, however, to the best of our knowledge, whether combination therapy reduces VAP occurrence during mechanical ventilation has not previously been studied $(12,13)$.

In the present study the sedative effects of dexmedetomidine combined with propofol in patients in the ICU undergoing mechanical ventilation were explored and the risk factors of VAP were assessed, in order to provide relevant references for clinicians.

\section{Materials and methods}

Patient data. The present study is a retrospective analysis of 322 patients that received mechanical ventilation in the ICU ward of Shanghai Fengxian District Central Hospital from May 2016 to June 2018. According to the use of anesthetics, patients were divided into a combined and monotherapy group. A total of 212 patients were included in the combined group, including 130 males and 82 females, and 110 patients were included in the monotherapy group, including 59 males and 51 females. The current study was approved by the Medical Ethics Committee of Shanghai Fengxian District Central Hospital and the family members of patients signed informed consent forms.

Inclusion and exclusion criteria. The patient inclusion criteria were as follows: i) $>18$ years old; ii) required mechanical ventilation according to a clinician's diagnosis; iii) complete clinical data; iv) mechanical ventilation time $<12 \mathrm{~h}$ or with an estimated mechanical ventilation time of $>24 \mathrm{~h}$; v) received sedative adjuvant therapy. Patient exclusion criteria were as follows: i) Pregnancy; ii) died within 3 days of mechanical ventilation or were transferred to other hospital within 3 days of mechanical ventilation; iii) had undergone digestive tract surgery or had a medical history of digestive disease, immune deficiency, central nervous system disease or cognitive impairment; iv) required renal replacement therapy; v) heart rate $<55$ beats/min.

Diagnostic criteria for VAP. Patients were diagnosed with VAP if they met the Guidelines for the Diagnosis and Treatment of Hospital-acquired Pneumonia at the Society of Respiratory Diseases of the Chinese Medical Association (14), but also met one of the following laboratory testing conditions: i) Leukocyte counts $>1^{*} 10^{11} / 1$; ii) leukocyte counts $<4^{*} 10^{10} / 1$; iii) body temperature $>37.5^{\circ} \mathrm{C}$; iv) purulent secretions in respiratory tract; $\mathrm{v}$ ) isolation of pathogenic bacteria from the airway.

Sedation schemes. Both groups were treated with fentanyl (Jiangsu Nhwa Pharmaceutical Co., Ltd.; cat. no. H20143315; $0.3 \mu \mathrm{g} / \mathrm{kg} / \mathrm{h}$ ) for continuous intravenous analgesia. In the combined group, dexmedetomidine (Jiangsu Hengrui Medicine Co., Ltd; cat. no. H20090248; $0.5 \mu \mathrm{g} / \mathrm{kg}$ ) was injected $10 \mathrm{~min}$ prior to fentanyl administration and continuously injected through a micro-injection pump (0.1-0.2 $\mathrm{mg} / \mathrm{kg} / \mathrm{h})$. Propofol (0.1-0.3 $\mathrm{mg} / \mathrm{kg} / \mathrm{h})$ was also continuously pumped intravenously. In the monotherapy group, dexmedetomidine $(0.5 \mu \mathrm{g} / \mathrm{kg})$ was slowly injected 10 min prior to fentanyl administration. Dexmedetomidine $(0.3-0.6 \mathrm{mg} / \mathrm{kg} / \mathrm{h})$ was continuously intravenously injected through a micro-injection pump.

Pathogenic bacteria cultures. An aseptic sputum aspirator was used to collect lower respiratory tract secretions and pharyngeal swabs were used to collect pharyngeal wall specimens every $24 \mathrm{~h}$. Pathogenic bacteria were detected using a BacT/Alert 3d60 automatic bacterial/mycobacterial culture monitoring system (Institut Mérieux).

Observation indicators. The main observation indicator was the number of patients with VAP in both the monotherapy and the combined group. According to VAP occurrence, patients were divided into VAP and non-VAP groups. Independent risk factors for the development of VAP were analyzed using multivariate analysis, and significance indicators were plotted using receiver operating characteristic (ROC) curves.

Secondary observation indicators, including clinical data such as sex, age, body mass index, past medical history, alcohol abuse, smoking history and acute physiology and chronic health evaluation II (APACHE II) scores (15) as well as sedative effects (onset time, waking time and extubation time), were compared between the groups. After extubation, the Ramsay scores (16) of the two groups were compared. The patients were scored as follows: A score of 1 was given if the patient was anxious, restless or, uneasy; a score of 2 was given if the patient was quiet, cooperative and had good directional force; a score of 3 was given if the patient was quiet and only responded to instructions; a score of 4 was given if the patient was in a state of sleep and only responding to light elastic stimulation between the eyebrows; a score of 5 was given if the patient was in a state of sleep and the response to light elastic stimulation between the eyebrows was slow; a score of 6 was given if the patient was in a state of sleep and did not respond to stimulation.

Statistical analysis. SPSS 20.0 software was used to analyze the collected data. GraphPad Prism 7 (GraphPad Software, Inc.) was used for image processing. Counting data were expressed as number (percentage) and assessed through the $\chi^{2}$ method. Kolmogorov-Smirnov tests were used to analyze data distributions. Measurement data were expressed as the mean \pm standard deviation. Independent sample t-tests were used to compare normal distribution data between the two groups. Using VAP as an independent variable and indicators with differences in a single factor as dependent variables, the independent risk factors of VAP were analyzed using the forward stepwise (Wald) method of multi-factor logistic regression. ROC curves were drawn to analyze indicators that had differences in multiple factors. $\mathrm{P}<0.05$ was considered to indicate a statistically significant difference.

\section{Results}

Comparison of clinical data. Through comparison of the clinical data between the groups, no significant differences in sex, age, body mass index, past medical history, history of alcoholism, history of smoking, residence and APACHE II scores were observed $(\mathrm{P}>0.05$; Table I). 
Table I. Clinical information.

\begin{tabular}{|c|c|c|c|c|}
\hline Characteristic & Monotherapy group $(n=110)$ & Combined group $(n=212)$ & $\chi^{2} / \mathrm{t}$ & P-value \\
\hline Sex & & & 1.764 & 0.184 \\
\hline Male & $59(53.64)$ & $130(61.32)$ & & \\
\hline Female & $51(46.36)$ & $82(38.68)$ & & \\
\hline Age & $57.20(7.30)$ & $59.00(8.40)$ & 1.842 & 0.067 \\
\hline BMI $\left(\mathrm{kg} / \mathrm{m}^{2}\right)$ & $22.44(1.55)$ & $22.69(1.95)$ & 1.167 & 0.244 \\
\hline \multicolumn{5}{|l|}{ Past medical history } \\
\hline Hypertension & $27(24.55)$ & $60(28.30)$ & 0.518 & 0.472 \\
\hline Diabetes & $12(10.91)$ & $27(12.74)$ & 0.227 & 0.634 \\
\hline COPD & $16(14.55)$ & $35(16.51)$ & 0.210 & 0.647 \\
\hline History of alcoholism & & & 1.179 & 0.278 \\
\hline Yes & $10(9.09)$ & $28(13.21)$ & & \\
\hline No & $100(90.91)$ & $184(86.79)$ & & \\
\hline History of smoking & & & 0.151 & 0.698 \\
\hline Yes & $65(59.09)$ & $130(61.32)$ & & \\
\hline No & $45(40.91)$ & $82(38.68)$ & & \\
\hline Residence & & & 0.348 & 0.555 \\
\hline City & $69(62.73)$ & $140(66.04)$ & & \\
\hline Country & $41(37.27)$ & $72(33.96)$ & & \\
\hline APACHE II score & $28.36(4.25)$ & $27.86(5.22)$ & 0.939 & 0.323 \\
\hline
\end{tabular}

Data are presented as (mean \pm standard deviation) or frequency (\%). BMI, body mass index; COPD, chronic obstructive pulmonary disease; APACHE, acute physiology and chronic health evaluation II.

Comparison of sedative effects. No significant differences in onset times were observed, but the waking and extubation times of the combined group were shorter than those of the monotherapy group. After extubation, the Ramsay score of the combined group was lower than that of the monotherapy group (Table II).

Occurrence of VAP and the distribution of pathogenic bacteria. A total of 54 patients with VAP were identified, 30 in the monotherapy group (27.27\%) and 24 in the combined group (11.32\%). The incidence of VAP was significantly higher in the monotherapy group $\left(\chi^{2}=16.565\right.$; $P=0.003$; Table III). According to the distribution of pathogenic bacteria detected through microorganisms, 72 pathogenic bacteria were detected in 54 patients. Amongst them, 38 strains (52.78\%) were Gram-negative bacteria, 32 strains $(44.44 \%)$ were Gram-positive and 2 strains (2.78\%) were fungi (Table IV).

Univariate analysis of patients with VAP. Patients were re-grouped into VAP $(n=54)$ and non-VAP groups $(n=268)$. Univariate analysis of their clinical data revealed no differences in sex, body mass index, past medical history, history of alcoholism, history of smoking, residence or onset time $(\mathrm{P}>0.05)$, whilst differences in age, APACHE II score, consciousness disorders, invasive operations, sedation programs, waking time, and extubation time were significant $(\mathrm{P}<0.001$; Table V).

Multivariate analysis and ROC curves. Single factors with significance were included and assigned (Table VI) for multivariate logistic regression analysis. Age, APACHE II score, consciousness disorders, invasive operations, waking time, extubation time and sedation regimens were independent risk factors for VAP in ICU patients undergoing mechanical ventilation (Table VII). Subsequently, ROC curves were plotted for the indicators of significant difference. The results demonstrated that the area under the curve of age, APACHE II score, consciousness disorders, invasive operations, waking time, extubation time and sedation regimens were $0.934,0.870,0.632,0.677,0.865,0.950$ and 0.603 , respectively (Fig. 1 and Table VIII).

\section{Discussion}

The ICU treats an array of critically ill patients (17). Due to their serious condition, many patients in the ICU have difficulties breathing. In the clinic, mechanical ventilation can assist breathing to keep the airways open, improving ventilation and oxygenation, to prevent hypoxia and carbon dioxide accumulation (18). However, patients in the ICU often suffer from intolerance to mechanical ventilation, as a result, sedatives are commonly used for assisted ventilation (19). The side effects of opioid use in the ICU include prolonged ventilation, psychiatric effects (such as irritability or hallucination), asceticism and urinary retention, which can reduce the patient compliance to treatment (20). According to the guidelines on analgesia, sedation and delirium issued by the American Society of Critical Care Medicine in 2013, patients in the ICU should try to treated to 'shallow sedation', where the patient is still responsive to language instructions, but where cognitive functions and 
Table II. Comparison of sedative effects.

\begin{tabular}{|c|c|c|c|c|}
\hline Group & Monotherapy group $(n=110)$ & Combined group $(n=212)$ & $\mathrm{t}$ value & P-value \\
\hline Onset time (s) & $16.25(4.88)$ & $17.54(6.24)$ & 1.889 & 0.060 \\
\hline Waking time (min) & $20.31(5.22)$ & $8.51(4.33)$ & 20.349 & $\mathrm{P}<0.001$ \\
\hline Extubation time (h) & $7.84(0.59)$ & $5.36(0.38)$ & 39.977 & $\mathrm{P}<0.001$ \\
\hline Ramsay score after extubation & $3.35 \pm 0.68$ & $2.62 \pm 0.49$ & 11.054 & $\mathrm{P}<0.001$ \\
\hline
\end{tabular}

Data are presented as (mean \pm standard deviation).

Table III. Comparison of ventilator-associated pneumonia incidence.

\begin{tabular}{lccr}
\hline Group & Occurred & Not occurred & $\chi^{2}$ value \\
\hline Monotherapy group $(\mathrm{n}=110)$ & 30 & 80 & 16.565 \\
Combined group $(\mathrm{n}=212)$ & 24 & 188 & 0.003 \\
\hline
\end{tabular}

Table IV. Comparison of distribution of pathogenic bacteria in VAP patients.

\begin{tabular}{lrc}
\hline Pathogenic bacteria & $\mathrm{n}$ & Constituent ratio (\%) \\
\hline Gram-negative bacterium & & \\
$\quad$ Staphylococcus aureus & 27 & 37.50 \\
Staphylococcus epidermidis & 8 & 11.11 \\
Enterococcus & 3 & 4.17 \\
Gram-positive bacterium & & \\
Pseudomonas aeruginosa & 23 & 31.94 \\
Klebsiella pneumoniae & 4 & 5.56 \\
Escherichia coli & 3 & 4.17 \\
Acinetobacter baumannii & 2 & 2.78 \\
Fungus & & \\
Candida albicans & 1 & 1.39 \\
Candida tropicalis & 1 & \\
\hline
\end{tabular}

VAP, ventilator-associated pneumonia.

coordination abilities are affected, without impacting on the breathing and circulation. Regardless of the sedation duration, dexmedetomidine or propofol are recommended for sedation, whilst benzodiazepines are not recommended (21).

As a common short-acting intravenous anesthetic, propofol provides anesthesia and sedation by acting on GABA receptors. However, propofol has clear inhibitory effects on the circulation and respiration, leading to lower blood pressure and slower heart rates in patients (22). As a highly selective $\alpha 2$ receptor agonist, dexmedetomidine has analgesic, sedative and anti-anxiety effects by promoting the secretion of GABA; compared with propofol, dexmedetomidine was not observed not cause respiratory depression, which was conducive to extubation (23). The sedative effects of dexmedetomidine used alone or in combination with propofol were compared in the present study. The results indicated that, although the dose of the two drugs was lower in the combined group, the two groups had similar onset times of anesthesia with the combined group showing significantly shorter waking times and extubation times compared with the monotherapy group. In studies by Xia et al (24), the sedative effects of propofol and dexmedetomidine alone on mechanical ventilation in the ICU were compared. The recovery and extubation times following dexmedetomidine treatment alone were significantly shorter than those of propofol. In the present study, shorter recovery and extubation times in response to the combination of dexmedetomidine and propofol were also observed, suggesting a good sedative effect. It can be speculated that the reason for this may be that the dose of the two drugs is reduced compared with that of the single drug, resulting in a shortening of the recovery time.

VAP is a common complication of mechanical ventilation in the ICU. The incidence of VAP in ICU patients after mechanical ventilation is high, which not only aggravates the condition, but also significantly increases mortality rates, which prolongs ICU residence times and increases costs $(22,23)$. The incidence of VAP was compared between monotherapy and combined groups and a significantly lower incidence of VAP was observed in the combined group compared with the monotherapy group, indicating that the sedation impacted the occurrence of VAP after mechanical ventilation in the ICU. However, to the best of our knowledge, no relevant studies on different sedation regimens as independent factors for VAP have been performed. In the present study clinical data was collected and were patients divided into VAP and non-VAP groups. Multivariate analysis indicated that age, APACHE II score, the disturbance of consciousness, invasive operations, waking time, extubation time and sedation programs were independent factors affecting VAP after mechanical ventilation in ICU patients. With increased patient age, body functions gradually weaken, and older patients have more basic diseases, such as hypertension, hyperlipidemia and diabetes, which often aggravates the condition (25). This is likely to be the main reason for the increased incidence of VAP after mechanical ventilation. 
Table V. Univariate analysis.

\begin{tabular}{|c|c|c|c|c|}
\hline Factor & VAP group $(n=54)$ & Non-VAP group $(n=268)$ & $\chi^{2} / \mathrm{t}$ value & P-value \\
\hline Sex & & & 2.977 & 0.084 \\
\hline Male & $26(48.15)$ & $163(60.82)$ & & \\
\hline Female & $28(51.85)$ & $105(39.18)$ & & \\
\hline Age & $65.54(3.25)$ & $58.41(3.65)$ & 13.326 & $\mathrm{P}<0.001$ \\
\hline $\operatorname{BMI}\left(\mathrm{kg} / \mathrm{m}^{2}\right)$ & $22.54(1.52)$ & $22.69(1.84)$ & 0.561 & 0.575 \\
\hline \multicolumn{5}{|l|}{ Past medical history } \\
\hline Hypertension & $13(24.07)$ & $74(27.61)$ & 0.182 & 0.671 \\
\hline Diabetes & $8(14.81)$ & $31(11.57)$ & 0.445 & 0.505 \\
\hline COPD & $10(18.52)$ & $41(15.30)$ & 0.350 & 0.554 \\
\hline History of alcoholism & & & 0.084 & 0.772 \\
\hline Yes & $7(12.96)$ & $31(15.57)$ & & \\
\hline No & $47(87.04)$ & $237(88.43)$ & & \\
\hline History of smoking & & & 1.013 & 0.314 \\
\hline Yes & $36(66.67)$ & $159(59.33)$ & & \\
\hline No & $18(33.33)$ & $109(40.67)$ & & \\
\hline Place of residence & & & 0.850 & 0.357 \\
\hline City & $38(70.37)$ & $171(63.81)$ & & \\
\hline Country & $16(29.63)$ & $97(36.19)$ & & \\
\hline APACHE II score & $31.55(2.58)$ & $25.33(3.84)$ & 11.389 & $\mathrm{P}<0.001$ \\
\hline Disturbance of consciousness & & & 22.381 & $\mathrm{P}<0.001$ \\
\hline Yes & $22(40.74)$ & $38(13.67)$ & & \\
\hline No & $32(59.26)$ & $240(86.33)$ & & \\
\hline Invasive operation & & & 30.719 & $\mathrm{P}<0.001$ \\
\hline Yes & $29(53.70)$ & $49(18.28)$ & & \\
\hline No & $25(46.30)$ & $219(81.72)$ & & \\
\hline Sedation scheme & & & 19.822 & $\mathrm{P}<0.001$ \\
\hline Monotherapy & $30(55.56)$ & $70(25.18)$ & & \\
\hline Combined therapy & $24(44.44)$ & $208(74.82)$ & & \\
\hline Onset time (sec) & $17.25(4.48)$ & $18.54(5.44)$ & 1.634 & 0.103 \\
\hline Recovery time (min) & $18.54(4.25)$ & $12.58(3.98)$ & 9.925 & $\mathrm{P}<0.001$ \\
\hline Extubation time (h) & $8.54(0.74)$ & $5.32(1.25)$ & 18.281 & $\mathrm{P}<0.001$ \\
\hline
\end{tabular}

Data are presented as mean \pm standard deviation), or frequency (\%). VAP, ventilator-associated pneumonia; BMI, body mass index; COPD, chronic obstructive pulmonary disease; APACHE, acute physiology and chronic health evaluation.

Table VI. Logistic regression factor assignment table.

Factor

Age (X)

APACHE scores (X)

Conscious disturbance (X)

Invasive operation

Onset time (X)

Extubation time (X)

Sedation scheme $(\mathrm{X})$

VAP situation (Y) $\mathrm{n}$

$<50$ years old $=1,51-55$ years old $=2,56-60$ years old $=3,>61$ years old $=4$ Using original data as it belongs to continuous variable

$\mathrm{Yes}=1, \mathrm{No}=2$

Yes $=1, \mathrm{No}=2$

Using original data as it belongs to continuous variable

The average extubation time is $5.85 ; \leq 5.85=1,>5.85=2$

Monotherapy $=1$, combined therapy $=2$

VAP patient $=1$, non-VAP patient $=2$

$\mathrm{X}$, independent variable; Y, dependent variable. VAP, ventilator-associated pneumonia; APACHE, acute physiology and chronic health evaluation. 
Table VII. Multivariate logistic regression analysis.

$95 \%$ CI of $\operatorname{Exp}(\beta)$

\begin{tabular}{lrrrrrrr} 
Factor & $\beta$ & SEM & Wals & P-value & Exp $(\beta)$ & Low limit & Upper limit \\
\hline Age & -2.172 & 0.571 & 14.459 & $<0.001$ & 0.114 & 0.037 & 0.349 \\
APACHE II scores & -0.449 & 0.130 & 11.889 & 0.001 & 0.638 & 0.494 & 0.824 \\
Disorder of consciousness & 2.630 & 1.066 & 6.090 & 0.014 & 13.870 & 1.718 & 111.973 \\
Invasive operation & 2.950 & 1.003 & 8.655 & 0.003 & 19.099 & 2.677 & 136.289 \\
Waking time & -0.302 & 0.128 & 5.571 & 0.018 & 0.740 & 0.576 & 0.950 \\
Extubation time & -5.451 & 1.540 & 12.394 & $<0.001$ & 0.004 & 0.000 & 0.089 \\
Sedation scheme & 1.825 & 0.818 & 4.983 & 0.026 & 6.204 & 1.249 & 30.805
\end{tabular}

APACHE, acute physiology and chronic health evaluation; Exp, expectation; CI, confidence interval.

Table VIII. ROC parameters.

\begin{tabular}{lcccccc}
\hline Parameter & AUC & $95 \%$ CI & Specificity $\%$ & Sensitivity $\%$ & Youden index \% & Cut-off \\
\hline Age & 0.934 & $0.904-0.964$ & 92.59 & 80.59 & 73.19 & $<61.00$ \\
APACHE II scores & 0.870 & $0.826-0.915$ & 79.63 & 80.59 & 60.23 & $<29.00$ \\
Disorder of consciousness & 0.632 & $0.545-0.721$ & 59.26 & 85.82 & 26.56 & 1 \\
Invasive operation & 0.677 & $0.592-0.762$ & 53.70 & 81.72 & 35.42 & 1 \\
Waking time & 0.865 & $0.814-0.915$ & 74.07 & 84.70 & 58.78 & $<16.47$ \\
Extubation time & 0.950 & $0.914-0.986$ & 94.44 & 92.54 & 86.98 & $<7.11$ \\
Sedation scheme & 0.603 & $0.518-0.687$ & 51.85 & 68.66 & 20.51 & 1
\end{tabular}

APACHE, acute physiology and chronic health evaluation; AUC, area under the curve; CI, confidence interval.

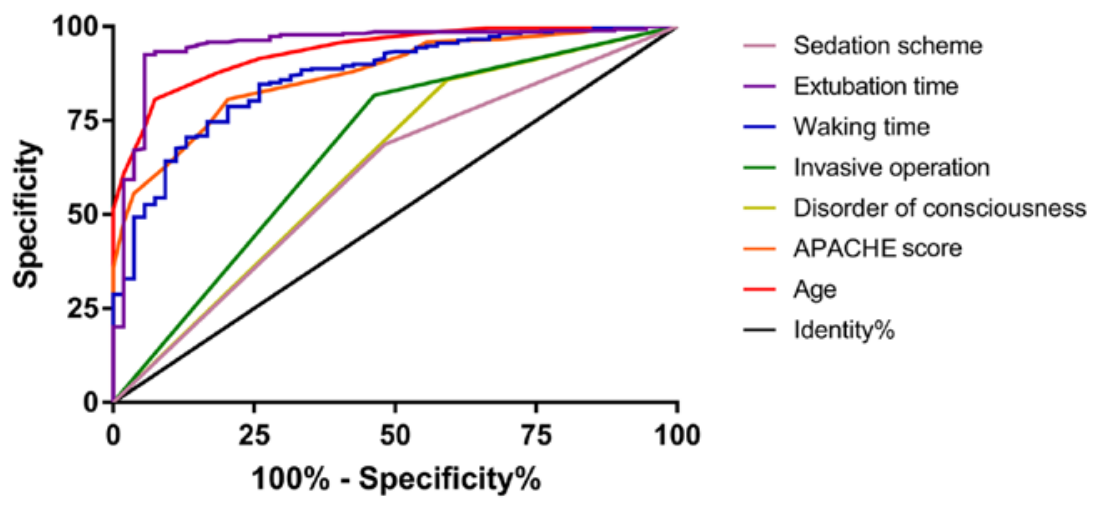

Figure 1. Receiver operating characteristic curve of ventilator-associated pneumonia occurrence, predicted by multi factor analysis difference index. ROC, receiver operating characteristic; APACHE, acute physiology and chronic health evaluation.

APACHE II scores are important comprehensive scores to assess the severity of the patients' condition. Higher scores indicate a higher risk of VAP. Invasive manipulation provides a direct channel for pathogens to enter the body. Moreover, invasive manipulations damage the respiratory mucosa and increase VAP incidence (11). In the different sedation schemes, the waking and extubation times of the monotherapy group were lower compared with the combined group. If the patient exhibited no consciousness disturbance after awakening, the machine was withdrawn ahead of schedule. Extubation times have been shown to be directly related to the incidence of VAP, mainly due to the fact tracheal insertion establishes a channel that increases infections by pathogenic bacteria infection. The shorter the extubation time, the lower the incidence of VAP (26).

The results of the present study indicated that a combination of drugs can shorten the waking and extubation times of patients with mechanical ventilation in the ICU. Multivariate analysis also suggests that different sedation schemes are independent risk factors for VAP. However, there are some 
limitations to the present study. As a retrospective analysis, selection bias can occur. Additionally patients treated with propofol only anesthesia were not included in the current study and their impact on the study findings requires assessment. Finally, statistics were not gathered regarding the adverse reactions of patients to the drugs. It is not clear whether the two schemes affect the adverse reactions of patients. Further prospective randomized controlled trials and other sedation regimens are now required to confirm these results.

In conclusion, dexmedetomidine combined with propofol may shorten the recovery and extubation time of patients with mechanical ventilation in the ICU compared with dexmedetomidine treatment alone. Different sedation schemes are also suggested to be independent risk factors for VAP in patients with mechanical ventilation in the ICU.

\section{Acknowledgements}

Not applicable.

\section{Funding}

No funding was received.

\section{Availability of data and materials}

The datasets use and/or anlayzed during the current study are available from the corresponding author on reasonable request.

\section{Authors' contributions}

HD was responsible for the research and analyzed the data; $\mathrm{FH}$ drafted the manuscript and analyzed the data.; WW and LL collected cases; DW and FL collected data.

\section{Ethics approval and consent to participate}

The present study was approved by the Medical Ethics Committee of Shanghai Fengxian District Central Hospital and all patients signed informed consent forms.

\section{Patient consent for publication}

Not applicable.

\section{Competing interests}

The authors declare that they have no competing interests.

\section{References}

1. Hu ZL, Zhang ZC, Li DW, Shuai WZ and Zou JF: Med J Chin People's Liberation Army 40: 6, 2015.

2. Jabaley CS, Groff RF, Sharifpour M, Raikhelkar JK and Blum JM: Modes of mechanical ventilation vary between hospitals and intensive care units within a university healthcare system: A retrospective observational study. BMC Res Notes 11: 425, 2018.

3. de Jong B, Schuppers AS, Kruisdijk-Gerritsen A, Arbouw ME, van den Oever HL and van Zanten AR: The safety and efficacy of nicotine replacement therapy in the intensive care unit: A randomised controlled pilot study. Ann Intensive Care 8: 70-70, 2018.
4. Sieber FE, Neufeld KJ, Gottschalk A, Bigelow GE, Oh ES, Rosenberg PB, Mears SC, Stewart KJ, Ouanes JP, Jaberi M, et al: Effect of depth of sedation in older patients undergoing hip fracture repair on postoperative delirium: The STRIDE randomized clinical trial. JAMA Surg 153: 987-995, 2018.

5. Aouad MT, Zeeni C, Al Nawwar R, Siddik-Sayyid SM, Barakat HB, Elias S and Yazbeck Karam VG: Dexmedetomidine for improved quality of emergence from general anesthesia: A dose-finding study. Anesth Analg 129: 1504-1511, 2019.

6. Weerink MA, Struys MM, Hannivoort LN, Barends CR, Absalom AR and Colin P: Clinical pharmacokinetics and pharmacodynamics of dexmedetomidine. Clin Pharmacokinet 56: 893-913, 2017.

7. Su X, Meng ZT, Wu XH, Cui F, Li HL, Wang DX, Zhu X, Zhu SN, Maze M and Ma D: Dexmedetomidine for prevention of delirium in elderly patients after non-cardiac surgery: A randomised, double-blind, placebo-controlled trial. Lancet 388: 1893-1902, 2016.

8. Qiu Q, Choi SW, Wong SS, Irwin MG and Cheung CW: Effects of intra-operative maintenance of general anaesthesia with propofol on postoperative pain outcomes-a systematic review and meta-analysis. Anaesthesia 71: 1222-1233, 2016.

9. Kreienbühl L, Elia N, Pfeil-Beun E, Walder B and Tramèr MR: Patient-controlled versus clinician-controlled sedation with propofol: Systematic review and meta-analysis with trial sequential analyses. Anesth Analg 127: 873-880, 2018.

10. Schurink CA, Nieuwenhoven CA, Jacobs JA, Rozenberg-Arska M, Joore HC, Buskens E, Hoepelman AI and Bonten MJ: Clinical pulmonary infection score for ventilator-associated pneumonia: Accuracy and inter-observer variability. Intensive Care Med 30: 217-224, 2004.

11. Karakuzu Z, Iscimen R, Akalin H, Kelebek Girgin N, Kahveci F and Sinirtas M: Prognostic risk factors in ventilator-associated pneumonia. Med Sci Monit 24: 1321-1328, 2018.

12. López-Aguilar J, Bassi GL, Quílez ME, Martí JD, Ranzani OT, Xiol EA, Rigol M, Luque N, Guillamat R, Ferrer I, et al: Hippocampal damage during mechanical ventilation in trendelenburg position: A secondary analysis of an experimental study on the prevention of ventilator-associated pneumonia. Shock 52: 75-82, 2019.

13. Louie J, Lonardo N, Mone M, Stevens V, Deka R, Shipley W and Barton R: 1576: Does the addition of dexmedetomidine to propofol sedation reduce duration of mechanical ventilation? Crit Care Med 46: 772, 2018.

14. Chinese Medical Association Respiratory Diseases Infectious Group: Guidelines for the diagnosis and treatment of acquired pneumonia and ventilator-associated pneumonia in Chinese adult hospitals (2018 edition. Chinese Journal of Tuberculosis and Respiratory Diseases (Zhong Hua Jie He He Hu Xi Za Zhi 4: 255-280, 2018

15. Qiu J, Wang C, Pan X, Pan L, Huang X, Xu J, Ji X and Mao M: APACHE-II score for anti-tuberculosis tolerance in critically ill patients: A retrospective study. BMC Infect Dis 19: 106, 2019.

16. Rasheed AM, Amirah MF, Abdallah M, P J P, Issa M and Alharthy A: Ramsay sedation scale and richmond agitation sedation scale: A cross-sectional study. Dimens Crit Care Nurs 38: 90-95, 2019.

17. Krinsley JS, Maurer P, Holewinski S, Hayes R, McComsey D, Umpierrez GE and Nasraway SA: Glucose control, diabetes status, and mortality in critically Ill patients: The continuum from intensive care unit admission to hospital discharge. Mayo Clin Proc 92: 1019-1029, 2017.

18. Dres M, Dubé BP, Mayaux J, Delemazure J, Reuter D, Brochard L, Similowski T and Demoule A: Coexistence and impact of limb muscle and diaphragm weakness at time of liberation from mechanical ventilation in medical intensive care unit patients. Am J Respir Crit Care Med 195: 57-66, 2017.

19. Venn RM and Grounds RM: Comparison between dexmedetomidine and propofol for sedation in the intensive care unit: Patient and clinician perceptions. Br J Anaesth 87: 684-690, 2001.

20. Rømsing J, Møiniche S, Mathiesen O and Dahl JB: Reduction of opioid-related adverse events using opioid-sparing analgesia with COX-2 inhibitors lacks documentation: A systematic review. Acta Anaesthesiol Scand 49: 133-142, 2005.

21. Barr J, Fraser GL, Puntillo K, Ely EW, Gélinas C, Dasta JF, Davidson JE, Devlin JW, Kress JP, Joffe AM, et al: Clinical practice guidelines for the management of pain, agitation, and delirium in adult patients in the intensive care unit. Crit Care Med 41: 263-306, 2013. 
22. Fogarty M, Kuck K, Orr JA, Sakata D, Brewer L, Johnson KB and Fang JC: Sa1040 a comparison of adequacy of ventilation with a non-invasive ventilator system vs. standard $\mathrm{O}_{2}$ with a nasal cannula for colonoscopy with moderate sedation using propofol and fentanyl. Gastrointest Endosc 85: AB164-AB165, 2017.

23. Singh G, Kaur H, Aggarwal S, Sharda G, Singh A, Jha A and Aggarwal H: Intravenous dexmedetomidine vs. lignocaine in attenuating the hemodynamic responses during laryngoscopy and endotracheal intubation: A randomized double blind study. Anaesthesia Pain Intensive Care 21: 181-186, 2017.
24. Xia ZQ, Chen SQ, Yao X, Xie CB, Wen SH and Liu KX: Clinical benefits of dexmedetomidine versus propofol in adult intensive care unit patients: A meta-analysis of randomized clinical trials. J Surg Res 185: 833-843, 2013.

25. Mulugeta W, Xue H, Glick M, Min J, Noe MF and Wang Y: Disease burdens and risk factors for diabetes, hypertension, and hyperlipidemia among refugees in Buffalo, New York, 2004-2014. J Health Care Poor Underserved 30: 1119-1131, 2019.

26. Isik A, Karavas E and Firat D: Spontaneous milk fistula from an axillary accessory breast. Breast J 25: 154, 2019. 